\title{
Gender differences in the presentation of chest pain in obstructive coronary artery disease: results from the Korean Women's Chest Pain Registry
}

\author{
Dong-Hyuk Cho ${ }^{1}$, Jimi Choi ${ }^{1}$, Mi-Na Kim ${ }^{1}$, Hack-Lyoung Kim², Yong Hyun $\mathrm{Kim}^{3}$, Jin Oh $\mathrm{Na}^{4}$, \\ Jin-Ok Jeong ${ }^{5}$, Hyun Ju Yoon ${ }^{6}$, Mi-Seung Shin ${ }^{7}$, Myung-A Kim² ${ }^{2}$ Kyung-Soon Hong ${ }^{8}$, Gil Ja Shin ${ }^{9}$, \\ Seong-Mi Park ${ }^{1}$, and Wan Joo Shim ${ }^{1}$
}

\begin{abstract}
Department of Internal Medicine, ${ }^{1}$ Korea University Anam Hospital, Seoul; ${ }^{2}$ Seoul Metropolitan Government Seoul National University Boramae Medical Center, Seoul; ${ }^{3}$ Korea University Ansan Hospital, Ansan; ${ }^{4}$ Korea University Guro Hospital, Seoul; ${ }^{5}$ Chungnam National University Hospital, Daejeon; ${ }^{6}$ Chonnam National University Hospital, Gwangju; ${ }^{7}$ Gachon University Gil Medical Center, Incheon; ${ }^{8}$ Hallym University Chuncheon Sacred Heart Hospital, Chuncheon; ${ }^{9}$ Ewha Womans University Mokdong Hospital, Seoul, Korea
\end{abstract}

Received: September 3, 2018 Revised : November 8, 2018 Accepted: November 20, 2018

\section{Correspondence to}

Wan Joo Shim, M.D.

Division of Cardiology,

Department of Internal

Medicine, Korea University

Anam Hospital, 73 Inchon-ro,

Seongbuk-gu, Seoul 02841, Korea

Tel: +82-2-920-5448

Fax: +82-2-927-1478

E-mail: wjshim@korea.ac.kr
Background/Aims: Chest pain in patients with obstructive coronary artery disease (OCAD) is affected by several social factors. The gender-based differences in chest pain among Koreans have yet to be investigated.

Methods: The study consecutively enrolled 1,549 patients (male/female, 514/1,035; $61 \pm 11$ years old) with suspected angina. The predictive factors for OCAD based on gender were evaluated.

Results: Men experienced more squeezing type pain on the left side of chest, while women demonstrated more dull quality pain in the retrosternal and epigastric area. After adjustment for risk factors, pain in the retrosternal area (odds ratio [OR], 1.491; 95\% confidence interval [CI], 1.178 to 1.887) and aggravation by exercise (OR, 2.235; 95\% CI, 1.745 to 2.861 ) were positively associated with OCAD. In men, shorter duration (OR, 1.581; 95\% CI, 1.086 to 2.303) and dyspnea (OR, 1.610; $95 \%$ CI, 1.040 to 2.490 ) increased the probability for OCAD, while left-sided chest pain suggested a low probability for OCAD (OR, 0.590; 95\% CI, 0.388 to 0.897 ). In women, aggravation by emotional stress (OR, 0.348; 95\% CI, 0.162 to 0.746 ) and dizziness (OR, $0.457 ; 95 \% \mathrm{CI}, 0.246$ to 0.849 ) decreased the probability for OCAD.

Conclusions: This is the first study to focus on gender differences in chest pain among Koreans with angina. Symptoms with high probability for OCAD were different between sexes. Our findings suggest that patient's medical history in pretest assessment for OCAD should be individualized considering gender.

Keywords: Chest pain; Sex characteristics; Coronary artery disease; Angina pectoris

\section{INTRODUCTION}

Chest pain is a common chief complaints and reason for referral to cardiology clinic. A wide range of diseases including coronary artery disease (CAD) may trigger chest pain. It is well known that careful history and identifying typical chest pain is the cornerstone of the diagnosis of CAD [1]. In most cases, it is possible to differentiate patients with CAD even based on the patient's history alone [2]. 
Angina is typically described as a squeezing and heavy sensation. The site of chest pain is usually retrosternal and the pain typically occurs under exertion or emotional stress; however, the typical description of angina is based on previous studies from Western countries [3-6]. Various factors including language, cultural syndrome and gender may contribute to the presentation of chest pain. Korea has its own culture and language, and the study about chest pain characteristics in Korean population is warranted. Furthermore, gender differences in the presentation of chest pain affect angina diagnosis and management [7-9]. However, chest pain characteristics of Koreans with angina, and symptoms suggestive of CAD, especially considering gender differences, have yet to be reported.

Therefore, our nation-wide, multicenter, prospective design study aims to evaluate whether gender difference exists in the description of angina symptoms. We further evaluated which symptoms are independently associated with the presence of obstructive coronary artery disease (OCAD) on coronary angiography (CAG).

\section{METHODS}

\section{Subjects}

The Korean Women's Chest Pain Registry (KoROSE) is a nation-wide, multicenter, prospective study evaluating patients who visited outpatient clinic with chest pain. The detailed protocol of the registry has been previously published $[10,11]$. The registration started from January 2012. The registry also enrolled men from February 2014 for comparison between sexes. Patients from 25 tertiary medical centers were registered until May 2018. Exclusion criteria were a history of myocardial infarction, or any coronary revascularization, or malignancy, or inflammatory diseases, or chronic kidney disease on dialysis. This study enrolled consecutive patients in the registry who underwent invasive CAG.

Clinical characteristics including age, gender, anthropometric parameters, and medical history of cardiovascular risk factors were investigated. Hypertension was defined by treatment with anti-hypertensive medications, and a systolic blood pressure (BP) $\geq 140$, or diastolic $\mathrm{BP} \geq 90 \mathrm{mmHg}$. Diabetes mellitus (DM) was defined by treatment with insulin or oral hypoglycemic agents or fasting blood glucose $\geq 126 \mathrm{mg} / \mathrm{dL}$. This study protocol was approved by the Institutional Review Boards of all participating centers (IRB No. 2012ANoo11). Written informed consent was obtained from all participants.

\section{Chest pain questionnaires}

Detailed chest pain questionnaires were obtained from all the patients by physicians. Six aspects of chest pain were evaluated as follows: (1) pain quality, (2) pain location, (3) pain duration, (4) aggravating factors, (5) location of radiating pain, and (6) associated symptoms. Pain quality was described as squeezing, pressure, and stinging, burning or dull pain. Patients were asked to point to painful locations on the body. The duration of pain was classified into three categories: shorter than 5 minutes, longer than 5 minutes and shorter than 15 minutes, longer than 15 minutes and shorter than 1 hour, and longer than 1 hour. Patients were asked to select the aggravating factors from the list: exercise, meal, emotional stress, low temperature, alcohol or other. The location of radiating pain was investigated among left arm, right arm, neck or back. Patients were also asked to indicate any associated symptoms from the list: palpitation, dizziness, headache, dyspnea, or syncope.

\section{Invasive coronary angiography}

To evaluate OCAD, invasive CAG was performed in each patient for the following indications: (1) typical angina, (2) angina with abnormal function test result, and (3) angina not evaluable with functional testing. Invasive CAG was performed via femoral or radial artery using a standard protocol. The percentage of coronary artery stenosis was determined by the ratio of the diameter of the stenotic lesion and normal site just proximal to the lesion. OCAD was defined as $\geq 70 \%$ stenosis in a proximal or middle epicardial coronary artery or major branch.

\section{Statistical analysis}

Categorical variables were presented with frequencies (percentages) and continuous variables were presented with mean \pm standard deviation. The differences in baseline and chest pain characteristics were compared between genders and according to the presence of OCAD using the independent $t$ test or chi-square test. Multivariable logistic regression was performed to investigate the impact of each chest pain characteristic for 
OCAD after adjustment for age, gender, hypertension, $\mathrm{DM}$, dyslipidemia, smoking, and family history of CAD. SPSS version 24 (IBM Co., Armonk, NY, USA) was utilized for statistical analysis. A $p<0.05$ was considered statically significant.

\section{RESULTS}

\section{Baseline characteristics of total population, men and women}

The study enrolled 1,549 consecutive patients (mean age $61.1 \pm 11.2$ years, 514 males and 1,035 females). Table 1 lists the clinical demographics of the total study population. The mean age of men was younger than that of women $(59.4 \pm 11.1$ years vs. $61.9 \pm 11.1$ years, $p<0.001)$. The prevalence of current smoking was higher in men compared

Table 1. Baseline characteristics of study population

\begin{tabular}{|c|c|c|c|c|}
\hline Characteristic & Total $(\mathrm{n}=1,549)$ & $\operatorname{Men}(n=514)$ & Women $(\mathrm{n}=1,035)$ & $p$ value $\mathrm{e}^{\mathrm{a}}$ \\
\hline Age & $61.1 \pm 11.2$ & $59.4 \pm 11.1$ & $61.9 \pm 11.1$ & $<0.001$ \\
\hline \multicolumn{5}{|l|}{ Cardiac risk factor } \\
\hline BMI, kg/m² & $25.1 \pm 3.5$ & $25.2 \pm 2.9$ & $25.0 \pm 3.7$ & 0.496 \\
\hline $\mathrm{BMI} \geq 25 \mathrm{~kg} / \mathrm{m}^{2}$ & $723(46.7)$ & $253(49.2)$ & $470(45 \cdot 4)$ & 0.087 \\
\hline Hypertension & $797(51.5)$ & $264(51.4)$ & $533(51.5)$ & 0.501 \\
\hline Diabetes mellitus & $311(20.1)$ & $104(20.2)$ & $207(20.0)$ & 0.482 \\
\hline Dyslipidemia & $305(19.7)$ & $92(17.9)$ & $213(20.6)$ & 0.118 \\
\hline Family history & $275(17.8)$ & $103(20.0)$ & $172(16.6)$ & 0.057 \\
\hline Current smoking & $210(13.6)$ & $167(32.5)$ & $43(4.2)$ & $<0.001$ \\
\hline \multicolumn{5}{|l|}{ Marital status } \\
\hline Married & $976(80.2)$ & $294(89.6)$ & $682(76.7)$ & $<0.001$ \\
\hline Single & $49(4 \cdot 0)$ & $17(5 \cdot 2)$ & $32(3.6)$ & 0.140 \\
\hline Divorced & $38(3.1)$ & $11(3.4)$ & $27(3.0)$ & 0.451 \\
\hline Widowed & $154(12.7)$ & $6(1.8)$ & $148(16.6)$ & $<0.001$ \\
\hline \multicolumn{5}{|l|}{ Occupational history } \\
\hline Production worker & $282(18.2)$ & $131(25 \cdot 5)$ & $151(14.6)$ & $<0.001$ \\
\hline Office worker & $229(14.8)$ & $138(26.8)$ & $91(8.8)$ & $<0.001$ \\
\hline None or housewife & $1,038(67.0)$ & $245(47.7)$ & $793(76.6)$ & $<0.001$ \\
\hline \multicolumn{5}{|l|}{ Medications } \\
\hline ACEi & $78(5.0)$ & $28(5.4)$ & $50(4.8)$ & 0.341 \\
\hline ARB & $400(25.8)$ & $135(26.3)$ & $265(25.6)$ & 0.412 \\
\hline $\mathrm{BB}$ & $320(20.7)$ & $96(18.7)$ & $224(21.6)$ & 0.098 \\
\hline $\mathrm{CCB}$ & $446(28.8)$ & $146(28.4)$ & $300(29.0)$ & 0.430 \\
\hline Diuretics & $106(6.8)$ & $32(6.2)$ & $74(7.1)$ & 0.286 \\
\hline Aspirin & $588(38.0)$ & $182(35 \cdot 4)$ & $406(39.2)$ & 0.080 \\
\hline Clopidogrel & $352(22.7)$ & $107(20.8)$ & $245(23.7)$ & 0.115 \\
\hline Statins & $661(42.7)$ & $225(43.8)$ & $436(42.1)$ & 0.286 \\
\hline
\end{tabular}

Values are presented as mean $\pm \mathrm{SD}$ or number (\%).

BMI, body mass index; ACEi, angiotensin converting enzyme inhibitor; ARB, angiotensin receptor blocker; BB, beta blocker; CCB, calcium channel blocker.

${ }^{\text {aT }}$ The result of independent $t$ test or chi-square test between men and women. 
Table 2. Chest pain characteristics of study population

\begin{tabular}{|c|c|c|c|c|}
\hline Characteristic & $\operatorname{Total}(\mathrm{n}=1,549)$ & $\operatorname{Men}(n=514)$ & Women $(n=1,035)$ & $p$ value $^{a}$ \\
\hline \multicolumn{5}{|l|}{ Pain quality } \\
\hline Squeezing & $568(36.7)$ & $207(40.3)$ & $361(34.9)$ & 0.022 \\
\hline Pressure & $396(25.6)$ & $128(24.9)$ & $268(25.9)$ & 0.361 \\
\hline Stinging & $261(16.8)$ & $93(18.1)$ & $168(16.2)$ & 0.197 \\
\hline Burning & $68(4 \cdot 4)$ & $20(3.9)$ & $48(4.6)$ & 0.297 \\
\hline Dull & $237(15.3)$ & $59(11.5)$ & $178(17.2)$ & 0.002 \\
\hline \multicolumn{5}{|l|}{ Pain location } \\
\hline Retrosternal & $817(52.7)$ & $256(49.8)$ & $561(54.2)$ & 0.057 \\
\hline Left side of chest & $368(23.8)$ & $160(31.1)$ & $208(20.1)$ & $<0.001$ \\
\hline Right side of chest & $47(3.0)$ & $17(3.3)$ & $30(2.9)$ & 0.382 \\
\hline Epigastric & $317(20.5)$ & $81(15.8)$ & $236(22.8)$ & 0.001 \\
\hline \multicolumn{5}{|l|}{ Pain duration } \\
\hline$<5 \min$ & $719(46.4)$ & $249(48.4)$ & $470(45.4)$ & 0.142 \\
\hline $5^{-15} \min$ & $281(18.1)$ & $99(19 \cdot 3)$ & $182(17.6)$ & 0.230 \\
\hline $15 \mathrm{~min}-1 \mathrm{hr}$ & $159(10.3)$ & $55(10.7)$ & $104(10.0)$ & 0.376 \\
\hline$>1 \mathrm{hr}$ & $390(25.2)$ & $111(21.6)$ & $279(27.0)$ & 0.012 \\
\hline \multicolumn{5}{|l|}{ Aggravating factors } \\
\hline Exercise & $449(29.0)$ & $161(31.3)$ & $288(27.8)$ & 0.086 \\
\hline Meal & $35(2.3)$ & $12(2.3)$ & $23(2.2)$ & 0.509 \\
\hline Stress & $122(7.9)$ & $36(7.0)$ & $86(8.3)$ & 0.214 \\
\hline Low temperature & $32(2.1)$ & $11(2.1)$ & $21(2.0)$ & 0.510 \\
\hline Alcohol & $10(0.6)$ & $6(1.2)$ & $4(0.4)$ & 0.074 \\
\hline \multicolumn{5}{|c|}{ Location of radiating pain } \\
\hline Left arm & $128(8.3)$ & $37(7.2)$ & $91(8.8)$ & 0.165 \\
\hline Right arm & $42(2.7)$ & $9(1.8)$ & $33(3.2)$ & 0.067 \\
\hline Neck & $99(6.4)$ & $32(6.2)$ & $67(6.5)$ & 0.473 \\
\hline Back & $148(9.6)$ & $29(5.6)$ & $119(11.5)$ & $<0.001$ \\
\hline \multicolumn{5}{|l|}{ Associated symptoms } \\
\hline Palpitation & $168(10.8)$ & $43(8.4)$ & $125(12.1)$ & 0.016 \\
\hline Dizziness & $164(10.6)$ & $55(10.7)$ & $109(10.5)$ & 0.491 \\
\hline Syncope & $27(1.7)$ & $9(1.8)$ & $18(1.7)$ & 0.566 \\
\hline Dyspnea & $369(23.8)$ & $115(22.4)$ & $254(24.5)$ & 0.190 \\
\hline Headache & $88(5.7)$ & $19(3.7)$ & $69(6.7)$ & 0.010 \\
\hline
\end{tabular}

Values are presented as number (\%).

${ }^{\text {a }}$ The result of chi-square test between men and women.

to women. However, other cardiovascular risk factors including hypertension, DM and dyslipidemia, and cardiovascular medications were not different between the genders.

\section{Chest pain characteristics}

Six chest pain characteristics including quality, duration, location, radiation, aggravation, and associated symptoms are listed in Table 2. Among all the enrolled patients, 568 patients (30.7\%) described squeezing qual- 
ity, and 817 patients $(52.7 \%)$ manifested pain in the retrosternal area. Pain duration shorter than 5 minutes was the most common (719 patients, $46.4 \%$ ), and was aggravated by exercise in 449 patients $(29.0 \%)$.

When chest pain characteristics were compared between genders, men experienced more squeezing chest pain, while women experienced more dull pain. Men complained more of left-sided chest pain, and women presented with epigastric pain. Pain duration exceeding one hour was more common in women than in men. More women described chest pain radiating to back with accompanying palpitation and headache.

\section{Chest pain related to OCAD}

OCAD on CAG was found in 425 patients (27.4\%).Chest pain was compared between patients with non-OCAD and OCAD (Table 3). Typical symptoms for angina were observed more in patients with OCAD compared with non-OCAD. In patients with OCAD, squeezing pain was more common than in patients with non-OCAD (171 [40.2\%] vs. 397 [35.3\%], $p=0.042$ ). Based on location, pain in the retrosternal area was predominant; however, left-sided pain in the chest was less common in patients with OCAD compared with non-OCAD (pain in retrosternal area: 251 [59.1\%] vs. 566 [50.4\%], $p=0.001$; left side of chest: 83 [19.5\%] vs. 285 [25.4\%], $p=0.009$ ). Duration of chest pain did not differ according to the presence of OCAD. Pain was aggravated by exercise more frequently, and less by emotional stress in patients with OCAD compared with non-OCAD (exercise: $175[41.2 \%]$ vs. 274 [24.4\%], $p<0.001$; emotional stress: 18 [4.2\%] vs. $104[9.3 \%], p<0.001)$. The location of radiating pain showed no difference according to the presence of OCAD. Among the associated symptoms, patients with OCAD complained of less palpitation and dizziness compared with patients with non-OCAD (palpitation: 26 [6.1\%] vs. 142 [12.6\%], $p<0.001$; dizziness: 26 [6.1\%] vs. $138[12.3 \%], p<0.001)$.

To investigate the independent association between each chest pain characteristic and OCAD, a multivariate logistic regression including age, gender, hypertension, $\mathrm{DM}$, dyslipidemia, smoking, and family history of CAD was performed (Table 4). The analysis revealed that pain in the retrosternal area and aggravation by exercise increased the probability of OCAD, while palpitation decreased the probability of OCAD (pain in retrosternal area: odds ratio [OR], 1.491; 95\% confidence interval [CI], 1.178 to $1.887, p=0.001$ ) (exercise: OR, 2.235; 95\% CI, 1.745 to $2.861, p<0.001$ ) (palpitation: OR, $0.476 ; 95 \% \mathrm{CI}, 0.304$ to $0.746 ; p=0.001$ ).

\section{Gender differences in chest pain characteristics and OCAD}

OCAD on CAG was more prevalent in men compared with women (175 [34.0\%] vs. 250 [24.2\%], $p<0.001)$. Men and women displayed differences in chest pain characteristics according to the presence of OCAD. In men, typical symptoms for angina were more commonly observed in patients with OCAD compared with non-OC$\mathrm{AD}$, while in women, a component of typical symptom was less commonly observed in patients with OCAD compared with non-OCAD. Quality of chest pain was not related to OCAD in both men and women. Left-sided chest pain was less commonly observed in men with OCAD compared with non-OCAD (42 [24.0\%] vs. 118 [34.8\%], $p=0.008)$. However, the location of chest pain was not related to OCAD in women. In terms of duration, only men demonstrated that shorter duration was related to OCAD (97 [55.4\%] vs. 152 [44.8\%], $p=0.014$ ). Among aggravation factors of chest pain, emotional stress was less frequent in women with OCAD compared with non-OCAD (8 [3.2\%] vs. 78 [9.9\%], p< 0.001), but the association was not significant in men. Among associated symptoms of chest pain, dyspnea was more common in men with OCAD compared with non-OC$\mathrm{AD}(49$ [28.0\%] vs. 66 [19.5\%], $p=0.019)$ and dizziness was less common in women with OCAD compared with non-OCAD (13 [5.2\%] vs. 96 [12.2\%], $p=0.001)$.

In men and women, multivariate logistic regression was performed to investigate the association between chest pain and OCAD after adjustment for age, hypertension, DM, dyslipidemia, smoking, and family history of CAD. Shorter duration of chest pain (less than 5 minutes) and dyspnea increased the probability of OCAD only in men (shorter duration: OR, 1.581; 95\% CI, 1.086 to 2.303; $p=0.017$ ) (dyspnea: OR, 1.610; 95\% CI, 1.040 to $2.490 ; p$ $=0.033)$. Left-sided chest pain decreased the probability of OCAD in men (OR, 0.590; $95 \%$ CI, 0.388 to $0.897 ; p=$ o.014), while aggravation by emotional stress and dizziness decreased the probability of OCAD in women (emotional stress: OR, $0.348 ; 95 \% \mathrm{CI}, 0.162$ to $0.746 ; p=0.007$ ) (dizziness: OR, 0.457 ; $95 \% \mathrm{CI}, 0.246$ to $0.849 ; p=0.013$ ). 


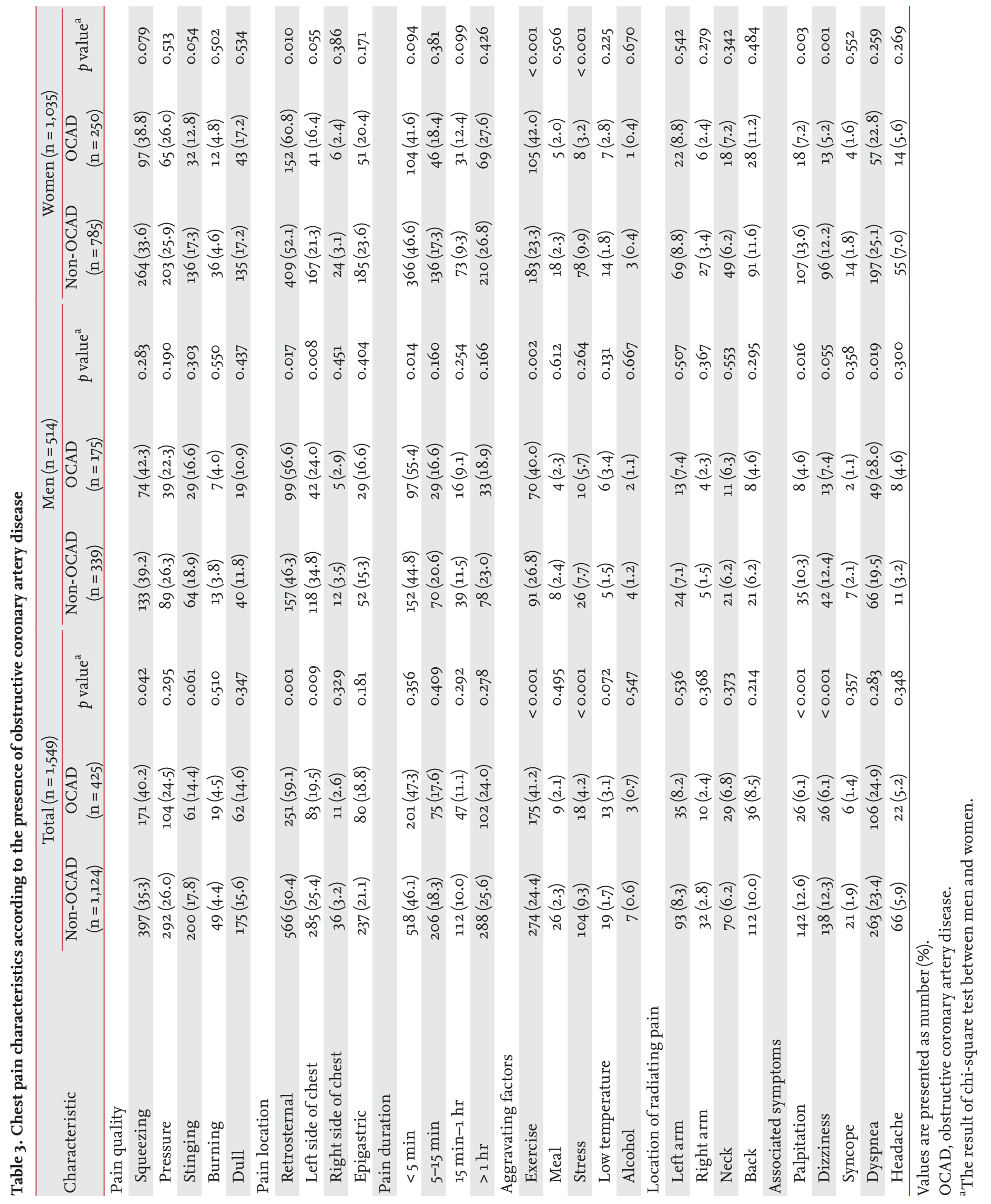




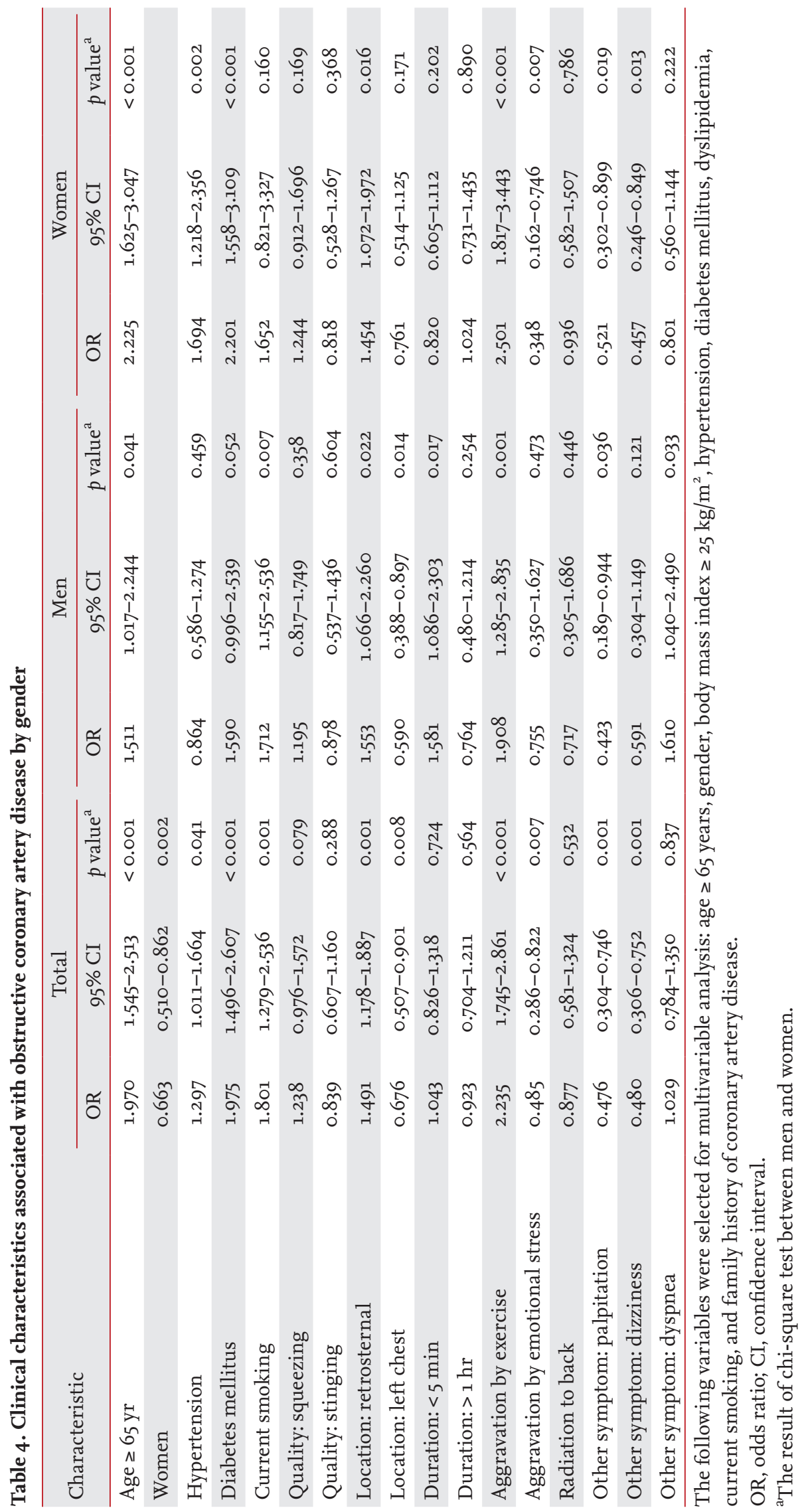




\section{DISCUSSION}

This is the first study to demonstrate gender differences in the presentation of chest pain and the risk for OCAD in Koreans with suspected angina. Our study demonstrated the following findings. (1) Men showed higher degrees of left-sided chest pain with a squeezing quality, while women presented with more dull pain in retrosternal and epigastric areas, radiating to the back, with palpitations and headache. (2) In both genders, pain in the retrosternal area and aggravation by exercise were independently associated with OCAD. (3) In men, duration shorter than 5 minutes and dyspnea increased the probability for OCAD, while left-sided chest pain had a low probability for OCAD. (4) In women, aggravation by emotional stress and dizziness lowered the probability for OCAD.

Our study has several strengths. This is a prospective study with a sufficient sample size to access gender differences in chest pain among patients with suspected angina in an outpatient setting. Furthermore, as a gold standard for the diagnosis of OCAD, invasive CAG was performed in all patients.

In patients with stable angina, women tend to be older than men, have more comorbidity such as hypertension, DM, dyslipidemia except for current smoking [12]. In this study, the mean age of women were older than men; however, other cardiovascular risk factors were well balanced between men and women except for current smoking. This population with relatively balanced risk factors between men and women could be an appropriate model to analyze gender difference of chest pain characteristics minimizing confounding factors.

\section{Chest pain and OCAD}

Stable angina is characterized by retrosternal squeezing, heaviness, and burning. It may radiate to arms, neck, jaw, and is triggered by exercise or emotional stress [2]. In the total study population, pain in retrosternal area and aggravation by exercise were associated with a high probability of OCAD. The results are compatible with the traditional description of stable angina. However, palpitation was negatively associated with OCAD. Palpitation is a common non-pain symptom [13]. However, the clinical implications of palpitation are unclear in patients with non- acute coronary syndrome. Our findings suggest that palpitation in patients with suspected angina may be an atypical symptom with a low probability of OCAD.

\section{Gender differences in chest pain}

Current practice guidelines in cardiovascular diseases (CVDs) are based on previous studies conducted primarily in men. For example, in the 2007 American Heart Association guidelines for the prevention of CVD in women, women constituted only $30 \%$ of all patients in clinical trials [14]. In a decade, numerous remarkable studies focused on clinical characteristics and outcomes of CVD in women [15-18]. In chest pain characteristics, gender differences have been reported variously according to study design (acute coronary syndrome or stable angina setting, patient population and methods to investigate chest pain characteristics). Although there are reports that chest pain characteristics were not significantly different between men and women with OCAD [19], women tend to have less specific and more non-cardiac symptoms compared with men $[7,8,13,20]$. Compared to men, CAD in women presents later in their lifespan. Because symptoms become less specific with aging, the differences in the distribution of age in men and women with CAD contribute to gender differences in chest pain presentation. The findings of current study that women older than men, and men presents more typical chest pain with squeezing quality and women manifest more dull pain and non-cardiac symptoms such as palpitation and headache is consistent with previous studies.

Although the gender differences in chest pain have been reported in patients with $\mathrm{CAD}$, their clinical implications and association with CAD are scarce. Majority of previous studies were performed in acute coronary syndrome setting, ant these studies demonstrated that diagnostic probability of chest pain characteristics are not significantly different between men and women $[21,22]$. In the current study among Koreans with stable chest pain in a tertiary care setting, the gender differences in symptoms for including or excluding CAD have been demonstrated.

Typically, a pain duration more than 30 minutes was the strong negative predictor for $\mathrm{CAD}$ [23]. In men from this study, shorter pain duration was positively associated with CAD. However, this association was not observed in women from this study. Similarly, the study 
with 1,212 German patients with chest pain in the primary care setting, also demonstrated that in men, shorter pain duration was positively associated with CAD, while pain duration exceeding 1 hour was associated with CAD in women [24]. Even though our findings were consistent with previous study, the underlying mechanism remains unclear.

In this study, only men showed left-sided chest pain was negatively associated with CAD. Bosner et al. [24] reported localized muscle tension in men was negatively associated with CAD. Because men tend to be more active in physical activity than women, it is possible that localized muscle tension, not CAD, may present as a left-sided chest pain. Simple physical examination such as chest wall tenderness on palpation is required to rule out the probability of CAD in these cases.

In men from this study, dyspnea was positively associated with CAD. The reason of association between dyspnea and $\mathrm{CAD}$ in men was not clear. But, smoking rate was much higher in men compared to women. As smoking is a potent common risk factor for both CAD and pulmonary disease, high smoking rate in men may be one cause of the association between dyspnea and CAD.

In women, aggravation by emotional stress was negatively associated with CAD. Although emotional stress is a traditional aggravation factor of angina [1], it is notable that in women from this study, pain worsening with emotional stress suggested a low probability of OCAD. In Western countries, somatization is more prevalent in women compared to men [25]. Higher frequency of depressive disorder in women also support the psychological etiology of chest pain in women [26]. Bosner et al. [24] also reported that in German population, women have more psychogenic disorder as the etiology of chest pain compared to men. Hwabyung is a Korean cultural syndrome and its prevalence in general population is $4.1 \%$ [27]. It is prevalent in middle-aged or older women. Chest pain in Korean women without OCAD may be explained as one of the somatization symptoms of Hwa-byung. The traditional notion that emotional stress is a predictor of CAD may be a misguided concept that has been derived from previous studies which had been conducted in population mainly composed of men rather than women. Further studies are needed to elucidate the psychological causes of chest pain in women.

\section{Underlying mechanisms for gender differences}

Currently, the underlying mechanism of gender difference in chest pain characteristics has not been clearly investigated; however, several mechanisms have been suggested. Firstly, gender differences in pain perception can be one mechanism. In positron emission tomography study, the gender difference of neurophysiological response by painful heat stimulation was observed and women perceive more intense pain than men [28]. In a population study, women tend to have more frequent and severe pain with more referring to greater portion of body region [29]. Second, coronary microvascular dysfunction is reported to be more common in women with suspected angina [30]. This difference of microvascular dysfunction may result in gender difference of chest pain characteristics. Lastly, on the inferior and posterior surface of heart, parasympathetic afferent nerves predominate while sympathetic nerves mainly innervate the anterior surface of heart. In women, right coronary dominance is more prevalent compared to men [31]. The proximal stenosis of a right coronary dominant artery may provoke myocardial ischemia on both anterior and posterior-inferior walls, and activate both sympathetic and parasympathetic nerve. Compared to men with a left coronary dominant system, women with right coronary artery stenosis have more probability to manifest autonomic symptoms such as nausea and emesis [31,32]. These anatomical and physiologic differences may explain the more observed autonomic symptoms such as epigastric pain, palpitation and dizziness in women of present study.

\section{Limitations}

Our study has several limitations. First, despite the growing concern about microvascular angina [33,34], our study did not investigate functional ischemia or microvascular dysfunction. We intend to conduct a coronary flow reserve test for additional patients enrolled. Our future study will investigate chest pain characteristics in patients with microvascular angina. Second, the number of men was relatively smaller than women in our study, suggesting a possible attenuation in the association between chest pain and OCAD in men. Finally, although this study suggested psychological causes as the non-cardiac factors in women's chest pain, our study did not conduct a psychological analysis. 
In conclusion, this is the first study to focus on gender differences in chest pain among Koreans with angina. Symptoms with high probability for OCAD varied between sexes. Our findings suggest that history taking in pretest assessment for OCAD should be individualized according to gender.

\section{KEY MESSAGE}

1. Although chest pain in patients with obstructive coronary artery disease (OCAD) is affected by several social factors, gender differences in the presentation of chest pain has not been evaluated in Koreans with suspected angina.

2. In men, duration shorter than 5 minutes and dyspnea increased the probability for OCAD, while left-sided chest pain had a low probability for OCAD.

3. In women, aggravation by emotional stress and dizziness lowered the probability for OCAD.

4. In pretest assessment for Korean patients with suspected angina, history taking should be individualized according to gender.

\section{Conflict of interest}

No potential conflict of interest relevant to this article was reported.

\section{Acknowledgments}

We appreciate the patients participating in this study.

\section{REFERENCES}

1. Task Force Members, Montalescot G, Sechtem U, et al. 2013 ESC guidelines on the management of stable coronary artery disease: the task force on the management of stable coronary artery disease of the European Society of Cardiology. Eur Heart J 2013;34:2949-3003.

2. Amsterdam EA, Kirk JD, Bluemke DA, et al. Testing of low-risk patients presenting to the emergency department with chest pain: a scientific statement from the American Heart Association. Circulation 2010;122:17561776.

3. Fox K, Garcia MA, Ardissino D, et al. Guidelines on the management of stable angina pectoris: executive summary: the task force on the management of stable angina pectoris of the European Society of Cardiology. Eur Heart J 2006;27:1341-1381.

4. Diamond GA. A clinically relevant classification of chest discomfort. J Am Coll Cardiol 1983;1:574-575.

5. Bosner S, Haasenritter J, Becker A, et al. Ruling out coronary artery disease in primary care: development and validation of a simple prediction rule. CMAJ 2010;182:12951300.

6. Skinner JS, Smeeth L, Kendall JM, Adams PC, Timmis A; Chest Pain Guideline Development Group. NICE guidance. Chest pain of recent onset: assessment and diagnosis of recent onset chest pain or discomfort of suspected cardiac origin. Heart 2010;96:974-978.

7. Mackay MH, Ratner PA, Johnson JL, Humphries KH, Buller CE. Gender differences in symptoms of myocardial ischaemia. Eur Heart J 2011;32:3107-3114.

8. Philpott S, Boynton PM, Feder G, Hemingway H. Gender differences in descriptions of angina symptoms and health problems immediately prior to angiography: the ACRE study. Appropriateness of Coronary Revascularisation study. Soc Sci Med 2001;52:1565-1575.

9. Regitz-Zagrosek V. Unsettled issues and future directions for research on cardiovascular diseases in women. Korean Circ J 2018;48:792-812.

10. Cho DH, Kim MA, Choi J, et al. Sex differences in the relationship between left ventricular diastolic dysfunction and coronary artery disease: from the Korean women's chest pain registry. J Womens Health (Larchmt) 2018;27:912-919.

11. Park SM, Kim MN, Kim SA, et al. Clinical significance of dynamic left ventricular outflow tract obstruction during dobutamine stress echocardiography in women with suspected coronary artery disease. Circ J 2015;79:2255-2262.

12. Hemal K, Pagidipati NJ, Coles A, et al. Sex differences in demographics, risk factors, presentation, and noninvasive testing in stable outpatients with suspected coronary artery disease: insights from the PROMISE trial. JACC Cardiovasc Imaging 2016;9:337-346.

13. D'Antono B, Dupuis G, Fortin C, Arsenault A, Burelle D. Angina symptoms in men and women with stable coronary artery disease and evidence of exercise-induced myocardial perfusion defects. Am Heart J 2006;151:813-819.

14. Wenger NK. Women and coronary heart disease: a century after Herrick: understudied, underdiagnosed, and 
undertreated. Circulation 2012;126:604-611.

15. Bairey Merz CN, Shaw LJ, Reis SE, et al. Insights from the NHLBI-sponsored Women's Ischemia Syndrome Evaluation (WISE) Study. Part II: gender differences in presentation, diagnosis, and outcome with regard to gender-based pathophysiology of atherosclerosis and macrovascular and microvascular coronary disease. J Am Coll Cardiol 2006;47:S21-S29.

16. Mieres JH, Gulati M, Bairey Merz N, et al. Role of noninvasive testing in the clinical evaluation of women with suspected ischemic heart disease: a consensus statement from the American Heart Association. Circulation 2014;130:350-379.

17. Mosca L, Benjamin EJ, Berra K, et al. Effectiveness-based guidelines for the prevention of cardiovascular disease in women: 2011 update: a guideline from the American Heart Association. J Am Coll Cardiol 2011;57:1404-1423.

18. Park SM, Merz CN. Women and ischemic heart disease: recognition, diagnosis and management. Korean Circ J 2016;46:433-442.

19. Kreatsoulas C, Shannon HS, Giacomini M, Velianou JL, Anand SS. Reconstructing angina: cardiac symptoms are the same in women and men. JAMA Intern Med 2013;173:829-831.

20. Granot M, Goldstein-Ferber S, Azzam ZS. Gender differences in the perception of chest pain. J Pain Symptom Manage 2004;27:149-155.

21. Devon HA, Rosenfeld A, Steffen AD, Daya M. Sensitivity, specificity, and sex differences in symptoms reported on the 13-item acute coronary syndrome checklist. J Am Heart Assoc 2014;3:e0oo586.

22. Rubini Gimenez M, Reiter M, Twerenbold R, et al. Sex-specific chest pain characteristics in the early diagnosis of acute myocardial infarction. JAMA Intern Med 2014;174:241-249.

23. Chun AA, McGee SR. Bedside diagnosis of coronary artery disease: a systematic review. Am J Med 2004;117:334-343.

24. Bosner S, Haasenritter J, Hani MA, et al. Gender differ- ences in presentation and diagnosis of chest pain in primary care. BMC Fam Pract 2009;10:79.

25. Wool CA, Barsky AJ. Do women somatize more than men? Gender differences in somatization. Psychosomatics 1994;35:445-452.

26. Marcus SM, Kerber KB, Rush AJ, et al. Sex differences in depression symptoms in treatment-seeking adults: confirmatory analyses from the Sequenced Treatment Alternatives to Relieve Depression study. Compr Psychiatry 2008;49:238-246.

27. Min SK. Hwabyung in Korea: culture and dynamic analysis. World Cult Psychiatry Res Rev 2009;4:12-21.

28. Paulson PE, Minoshima S, Morrow TJ, Casey KL. Gender differences in pain perception and patterns of cerebral activation during noxious heat stimulation in humans. Pain 1998;76:223-229.

29. Breivik H, Collett B, Ventafridda V, Cohen R, Gallacher D. Survey of chronic pain in Europe: prevalence, impact on daily life, and treatment. Eur J Pain 2006;10:287-333.

30. Marroquin OC, Holubkov R, Edmundowicz D, et al. Heterogeneity of microvascular dysfunction in women with chest pain not attributable to coronary artery disease: implications for clinical practice. Am Heart J 2003;145:628635 .

31. Arslanian-Engoren C, Engoren M. Physiological and anatomical bases for sex differences in pain and nausea as presenting symptoms of acute coronary syndromes. Heart Lung 2010;39:386-393.

32. Arora G, Bittner V. Chest pain characteristics and gender in the early diagnosis of acute myocardial infarction. Curr Cardiol Rep 2015;17:5.

33. Crea F, Bairey Merz CN, Beltrame JF, et al. The parallel tales of microvascular angina and heart failure with preserved ejection fraction: a paradigm shift. Eur Heart J 2017;38:473-477.

34. Park JJ, Park SJ, Choi DJ. Microvascular angina: angina that predominantly affects women. Korean J Intern Med 2015;30:140-147 